\title{
Numerical Study on Quantum Efficiency Enhancement of a Light-Emitting Diode Based on Surface Plasmon Coupling With a Quantum Well
}

\author{
Wen-Hung Chuang, Jyh-Yang Wang, C. C. Yang, and Yean-Woei Kiang
}

\begin{abstract}
We demonstrate the numerical study results of the enhancements of internal quantum efficiency (IQE) and external quantum efficiency (EQE) of a semiconductor quantum well when it is coupled with surface plasmons (SPs) induced on a grating interface between Ag and semiconductor. The IQE and EQE enhancements depend on the emission dipole position and the assigned intrinsic IQE. The SP dissipation in metal and the grazing-angle SP radiation lead to a significant difference between IQE and EQE. The enhancement of EQE is less significant when the intrinsic IQE becomes larger. In applying the SP coupling phenomenon to an InGaN-GaN quantum-well light-emitting diode, the efficiency enhancement is more significant in the green-red range, in which the intrinsic IQE is normally quite low.
\end{abstract}

Index Terms-Light-emitting diodes, quantum wells (QWs), surface waves.

$\mathbf{S}$ URFACE PLASMON (SP) coupling with a light emitter/ absorber has been widely implemented for enhancing emission/absorption efficiency. In particular, by coupling a light emitter with a bright SP mode, which can effectively radiate, the emission efficiency can be enhanced for practical device application [1]-[7]. In such a coupling process, the light emitter first transfers its energy into the SP mode through the coverage of the light emitter by the evanescent field of an SP mode. If the SP mode can radiate effectively, the coupling process can enhance not only the internal quantum efficiency (IQE), but also the light extraction efficiency of a light-emitting device. However, an SP mode may suffer from the high loss of metal dissipation. Also, the grazing-angle SP radiation is practically not useful that further degrades the external quantum efficiency (EQE) [7]. Therefore, it is important to evaluate the IQE and EQE of a light emitter, which couples with SP modes in a metal/dielectric interface geometry, before the coupling process can be applied to a practical device. In particular, the net contributions to IQE and $\mathrm{EQE}$ of a light emitter array, for simulating an excited quantum well (QW), in coupling with SP modes are interesting for the application to an $\mathrm{InGaN}-\mathrm{GaN}$ QW light-emitting diode.

Manuscript received March 18, 2008; revised May 15, 2008. This work was supported by the National Science Council, Republic of China, under Grants NSC 96-2120-M-002-008 and NSC 96-2221-E-002-188, and by the U.S. Air Force Scientific Research Office under Contract AOARD-07-4010.

The authors are with the Institute of Photonics and Optoelectronics and Department of Electrical Engineering, National Taiwan University, Taipei, 10617 Taiwan, R.O.C. (e-mail: ccy@cc.ee.ntu.edu.tw; ywkiang@ntu.edu.tw).

Color versions of one or more of the figures in this letter are available online at http://ieeexplore.ieee.org.

Digital Object Identifier 10.1109/LPT.2008.926853

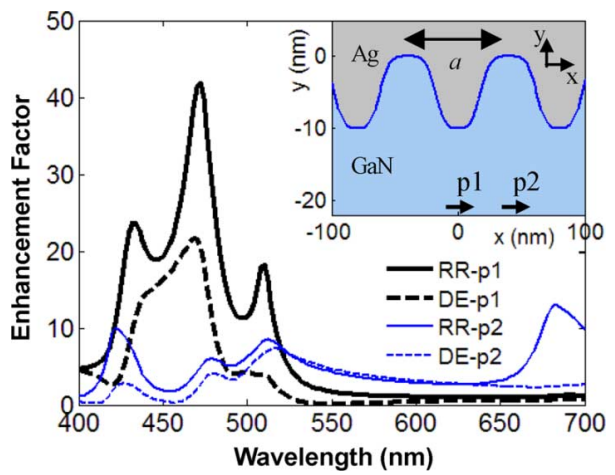

Fig. 1. Enhancement factor spectra of dipole radiation rate (RR) and detectable downward-propagating emission (DE) in the cases of dipoles $\mathrm{p} 1(-\mathrm{p} 1)$ and $\mathrm{p} 2$ $(-\mathrm{p} 2)$ when the grating period is $80 \mathrm{~nm}$. The structure geometry under study is shown in the inset.

In this letter, we report the numerical study results of IQE and EQE enhancements of a radiation dipole when it is coupled with SP modes generated on a grating interface between a half-space of Ag and a half-space of GaN. Because this research means to reveal the fundamental characteristics of SP coupling, the detailed device structure is not considered such that the device $\mathrm{EQE}$ cannot be evaluated. Instead, a quasi-EQE (QEQE) is defined to represent the emission efficiency after the factors of SP loss and SP grazing-angle radiation are taken into account. The dependencies of IQE and QEQE on the dipole position relative to the grating phase are illustrated. Based on the assumption of incoherent superposition, the average IQE and QEQE, including the contributions of multiple dipoles at different positions, are evaluated. The numerical calculations are performed using the plane-wave-assisted boundary integralequation method [8]. For computation, we discretize the unknown equivalent surface currents on all boundaries and interfaces by expanding them with the local linear bases. Then the Galerkin testing procedure is used to transfer the whole integral equation into a matrix equation.

Fig. 1 shows the enhancement factors of the dipole radiation rate and the downward-propagating emission [7] in a problem geometry shown in the inset. Here, a one-dimensional grating interface of $80 \mathrm{~nm}$ in period (denoted by a 80 ) divides the space into a half-space of $\mathrm{Ag}$ and a half-space of $\mathrm{GaN}$ (with a fixed refractive index at 2.5). The shape of grooves is described by a super-Gaussian function. The permittivity of $\mathrm{Ag}$ is assumed to follow the Drude model [9] with the angular plasma frequency set at $\omega_{p}=1.19 \times 10^{16}\left(\mathrm{rad} \cdot \mathrm{s}^{-1}\right)$ and damping constant set at $\gamma=1.33 \times 10^{14}\left(\mathrm{rad} \cdot \mathrm{s}^{-1}\right)$, leading to the surface plasmon polariton (SPP) resonance energy of $2.92 \mathrm{eV}(425 \mathrm{~nm}$ 


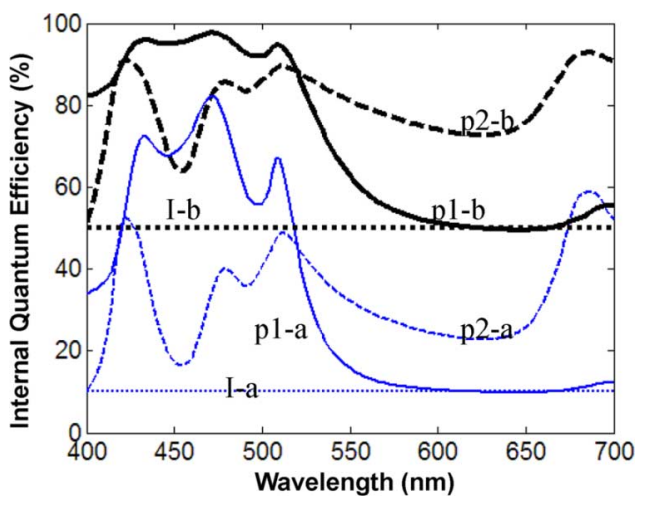

Fig. 2. IQEs as functions of wavelength for the two cases of constant dipole intrinsic IQE at $10 \%$ (I-a) and 50\% (I-b) with dipoles p1 (p1-a for the case of $\mathrm{I}-\mathrm{a}$ and $\mathrm{p} 1-\mathrm{b}$ for the case of I-b) and $\mathrm{p} 2$ (p2-a for the case of I-a and p2-b for the case of I-b).

in wavelength) at the plane $\mathrm{Ag}-\mathrm{GaN}$ interface. Two dipoles denoted by $\mathrm{p} 1$ and $\mathrm{p} 2$ are located at $10 \mathrm{~nm}$ right below a grating valley, with the coordinate at $(0,-20 \mathrm{~nm})$, and at $20 \mathrm{~nm}$ right below a crest of the grating, with the coordinate at $(40 \mathrm{~nm}$, $-20 \mathrm{~nm}$ ), respectively. The dipoles are extended infinitely in the $+z$ - and $-z$-directions to form a two-dimensional problem. Because Ag is infinitely thick, only the downward-propagating emission (denoted by DE in Fig. 1) is detectable. The definitions of IQE and QEQE are given by $\eta_{\text {int }}=P_{\text {rad }} /\left(P_{\text {rad }}+P_{\text {non }}\right)$ and $\eta_{\mathrm{qext}}=P_{\text {down }} /\left(P_{\mathrm{rad}}+P_{\mathrm{non}}\right)$, respectively. Here $P_{\mathrm{rad}}$ denotes the total radiated power by the dipole source, $P_{\text {down }}$ is the downward-propagating emission power, and $P_{\text {non }}$ is the power corresponding to the nonradiative recombination, which can be calculated for a given intrinsic IQE value.

In Fig. 1, the curves of RR-p1 and DE-p1 (RR-p2 and DE-p2) represent the enhancement factors of the dipole radiation rate and the downward-propagating emission, respectively, when dipole $\mathrm{p} 1$ (p2) is coupled with the generated SP modes on the grating. The differences between the RR and $\mathrm{DE}$ curves are attributed to the SP dissipation loss and the SP grazing-angle radiation. The three peaks in the case of $\mathrm{p} 1$ originate from the $\mathrm{SPP}$ on the plane $\mathrm{Ag}-\mathrm{GaN}$ interface (around $430 \mathrm{~nm}$ ), the localized surface plasmon (LSP) corresponding to the groove shape (around $470 \mathrm{~nm}$ ), and the SPP corresponding to the grating of $80 \mathrm{~nm}$ in period (around $510 \mathrm{~nm}$ ). In the case of $\mathrm{p} 2$, the aforementioned three peaks can still be seen even though their peak positions are slightly shifted and the relative intensities are changed. The lower LSP feature around $480 \mathrm{~nm}$ in the $\mathrm{p} 2$ case is due to the longer distance of $\mathrm{p} 2$ from the semiconductor groove of either side. The spectral shifts of the peaks show that the excited SP mode energy is dependent on the dipole position. In the $\mathrm{p} 2$ case, both radiation and emission enhancements show long tails on the long-wavelength side. There is a strong peak of radiation enhancement around $680 \mathrm{~nm}$. This feature of insignificant downward-propagating emission is related to a mixture of SPP and LSP modes and will be elucidated elsewhere.

Fig. 2 shows the evaluated IOEs corresponding to the problem geometry in Fig. 1. The IQE is evaluated by first assigning an intrinsic IQE to determine the nonradiative recombination rate. Then, the intrinsic radiative recombination rate is replaced by the radiation rate shown in Fig. 1 to obtain the IQE under the SP coupling condition. In Fig. 2, we consider two intrinsic IQE values (constants over the whole concerned spectral range) at

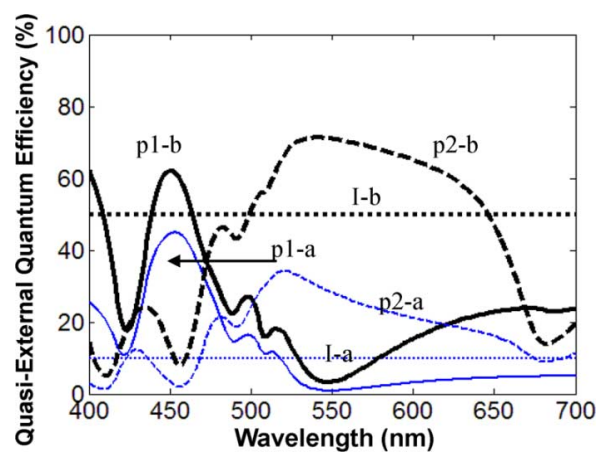

Fig. 3. QEQEs as functions of wavelength for the cases shown in Fig. 2.

$10 \%(\mathrm{I}-\mathrm{a})$ and $50 \%(\mathrm{I}-\mathrm{b})$. The IQEs of dipole $\mathrm{p} 1$ (p2) are shown in the curves of $\mathrm{p} 1-\mathrm{a}$ and $\mathrm{p} 1-\mathrm{b}$ (p2-a and $\mathrm{p} 2-\mathrm{b}$ ) when the intrinsic IQEs of I-a and I-b are considered, respectively. Here, one can see that the spectra of IQEs essentially follow the patterns of radiation rate enhancement in Fig. 1. Over 95\% IQE of dipole p1 in the blue-green range can be achieved when the intrinsic IQE is $50 \%$.

Fig. 3 shows the spectra of QEQEs corresponding to the cases in Fig. 2. The evaluation of a QEQE is similar to that of an IQE except that the enhanced radiation rate is replaced by the enhanced downward-propagating emission. Here, one can see that the SP coupling with dipole $\mathrm{p} 1$ can enhance the QEQE from $10 \%$ intrinsic IQE (the same as EQE if the light extraction is $100 \%$ ) up to around $45 \%$ (up to 4.5 times) in the blue range (around $460 \mathrm{~nm}$ ). Also, the SP coupling with dipole p2 can enhance the QEQE from $10 \%$ intrinsic IQE up to $35 \%$ (up to 3.5 times) in the green range (around $520 \mathrm{~nm}$ ). However, when the intrinsic IQE is increased to $50 \%$, only up to 1.5 times enhancement in QEQE can be achieved through the SP coupling. In other words, the SP-coupling-induced QEQE enhancement becomes less significant as the intrinsic IQE increases. This result is reasonable because a high intrinsic IQE or low nonradiative recombination rate implies that the photon emission channel through SP coupling does not necessarily help in emission because of the extra loss channels of SP dissipation and grazing-angle radiation.

From Figs. 2 and 3, one can see the different contributions of dipoles p1 and p2 to IQE and QEQE. Dipoles at different lateral positions are supposed to result in different levels of IQE and QEQE. Fig. 4 shows the IQE and QEQE at $460 \mathrm{~nm}$ with the intrinsic IQE at 50\% when the dipole is located at different $\mathrm{x}$-coordinates. Two grating periods of $80(a 80)$ and $108(a 108)$ $\mathrm{nm}$ are considered. Here, one can see that although the IQEs can always be enhanced up to $>70 \%$, only those dipoles located around the grating valleys can make the QEQE slightly higher than the intrinsic IQE. Fig. 5 shows the IQE and QEQE at $520 \mathrm{~nm}$ with the intrinsic IQE at $10 \%$ when the dipole is located at different $\mathrm{x}$-coordinates. In this situation, except those dipoles located around the grating valleys, QEQEs can always be higher than the intrinsic IQE in either case of grating period.

An array of dipoles in the plane of $y=-20 \mathrm{~nm}$ can be used for simulating a QW, which couples with the induced SP modes on the metallic grating. In such a coupling process, we may expect certain coherency when more than one dipole is coupled with the same SP mode. Coherent coupling of multiple dipoles is supposed to lead to stronger emission when compared with the 


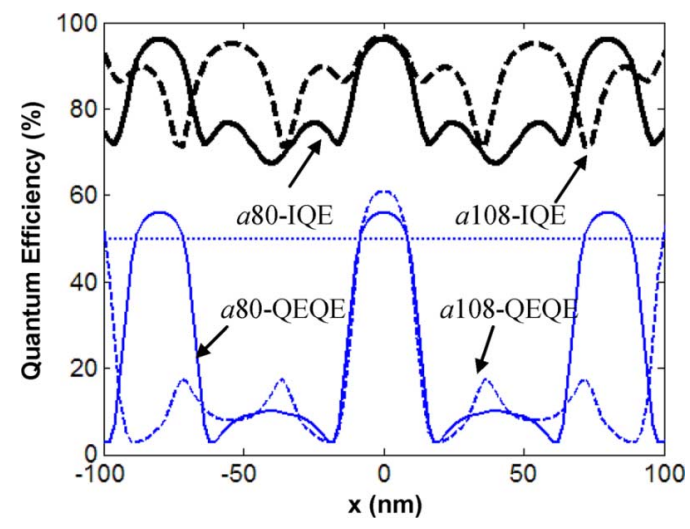

Fig. 4. IQEs and QEQEs at $460 \mathrm{~nm}$ as functions of dipole position $x$ when the intrinsic IQE is $50 \%$ (the horizontal dotted line) for the two cases of different grating periods at $80(a 80)$ and $108(a 108) \mathrm{nm}$.

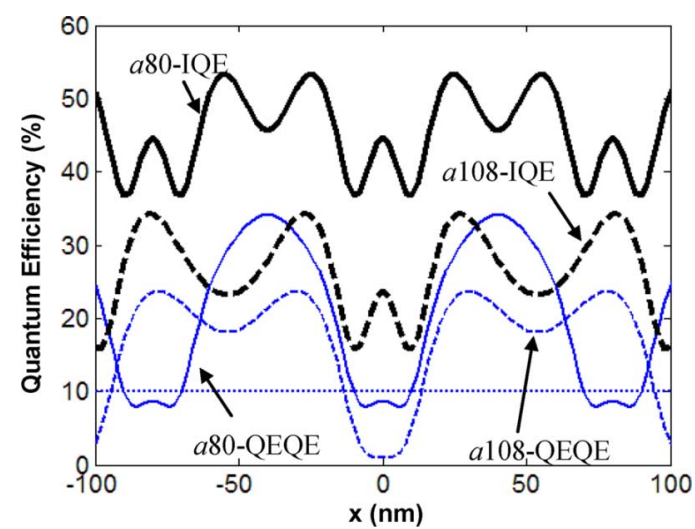

Fig. 5. IQEs and QEQEs at $520 \mathrm{~nm}$ as functions of dipole position $x$ when the intrinsic IQE is $10 \%$ (the horizontal dotted line) for the two cases of different grating periods at $80(a 80)$ and $108(a 108) \mathrm{nm}$.

TABLE I

Average IQE AND QEQE VAlues AT DIFFERENT GRATING PERIODS AND DIFFERENT INTRINSIC IQES

\begin{tabular}{cccc}
\hline Wavelength & $\mathbf{4 6 0} \mathbf{~} m$ & $\mathbf{5 2 0} \mathbf{n m}$ & $\mathbf{6 2 0} \mathbf{~ n m}$ \\
\hline Intrinsic IQE (\%) & 50 & 10 & 2 \\
\hline $\begin{array}{c}\text { a80 grating } \\
\text { IQE/QEQE (\%) }\end{array}$ & $79.03 / 21.61$ & $46 / 21.99$ & $3.89 / 2.91$ \\
\hline $\begin{array}{c}\text { a108 grating } \\
\text { IQE } \mathbf{Q E Q E}(\%)\end{array}$ & $87.93 / 19.10$ & $26.19 / 16.08$ & $9.12 / 4.34$ \\
\hline
\end{tabular}

completely incoherent emission of different dipoles in a QW. To simplify the discussion, we assume the completely incoherent emission among the dipoles to evaluate the lower bounds of IQE and QEQE. For numerical simulation, we use multiple incoherent dipoles equally spaced by $2 \mathrm{~nm}$ in the $\mathrm{x}$-axis to simulate a QW for evaluating the average IQE and QEQE. Table I lists the average IQE and QEQE values at different grating periods and different intrinsic IQEs. The intrinsic IQEs for different wavelengths are chosen to be consistent with the realistic situations of crystal growth. In this table, one can see that with the assumed grating conditions, the SP coupling does not help in emission enhancement in the blue range [10], [11]. However, because of the low intrinsic IQE in the green and red ranges, SP coupling can significantly enhance emission efficiency. It is noted that the assumed grating structure may not be optimized yet for the practical application because the LSP mode pattern, which determines whether it is a bright or dark mode, is sensitive to the metal shape. Compared with a grating structure, a nano-scale rough metal surface may be a better choice because it can provide various groove geometries for supporting various LSP and SPP modes [12]. Some of those SP modes may lead to a significant enhancement of emission efficiency.

In summary, we have demonstrated the numerical study results of IQE and QEQE of a dipole when it was coupled with the SP modes induced on a grating interface between $\mathrm{Ag}$ and $\mathrm{GaN}$. The IQE and QEQE enhancements depended on the dipole position and the assigned intrinsic IQE. The SP dissipation in metal and SP grazing-angle radiation led to a significant difference between IQE and QEQE. The enhancement of QEQE became weaker as the intrinsic IQE was increased. It is noted that in photoluminescence (PL) measurement of SP-QW coupling, PL intensity could be significantly enhanced in an InGaN-GaN QW sample of high intrinsic IQE. Such a result can be due to the Fabry-Pérot effect of the PL excitation laser.

\section{REFERENCES}

[1] A. Neogi, C. W. Lee, H. O. Everitt, T. Kuroda, A. Tackeuchi, and E. Yablonovitch, "Enhancement of spontaneous recombination rate in a quantum well by resonant surface plasmon coupling," Phys. Rev. B, vol. 66, no. 15, p. 153305, Oct. 2002.

[2] K. Okamoto, I. Niki, A. Shvartser, Y. Narukawa, T. Mukai, and A. Scherer, "Surface-plasmon-enhanced light emitters based on InGaN quantum wells," Nat. Mater., vol. 3, no. 9, pp. 601-605, Sep. 2004.

[3] C. Y. Chen, Y. C. Lu, D. M. Yeh, and C. C. Yang, "Influence of the quantum-confined Stark effect in an InGaN/GaN quantum well on its coupling with surface plasmon for light emission enhancement," Appl. Phys. Lett., vol. 90, no. 18, p. 183114, May 2007.

[4] Y. C. Lu, C. Y. Chen, D. M. Yeh, C. F. Huang, T. Y. Tang, J. J. Huang, and C. C. Yang, "Temperature dependence of the surface plasmon coupling with an InGaN/GaN quantum well," Appl. Phys. Lett., vol. 90, no. 19, p. 193103 , Apr. 2007.

[5] D. M. Yeh, C. F. Huang, Y. C. Lu, C. Y. Chen, T. Y. Tang, J. J. Huang, K. C. Shen, Y. J. Yang, and C. C. Yang, "Surface plasmon leakage in its coupling with an InGaN/GaN quantum well through an ohmic contact," Appl. Phys. Lett., vol. 91, no. 6, p. 063121, Aug. 2007.

[6] D. M. Yeh, C. F. Huang, C. Y. Chen, Y. C. Lu, and C. C. Yang, "Surface plasmon coupling effect in an InGaN/GaN single-quantum-well lightemitting diode," Appl. Phys. Lett., vol. 91, no. 17, p. 171103, Oct. 2007.

[7] J. Y. Wang, Y. W. Kiang, and C. C. Yang, "Emission enhancement behaviors in the coupling between surface plasmon polariton on a onedimensional metallic grating and a light emitter," Appl. Phys. Lett., vol. 91, no. 23, p. 233104, Dec. 2007.

[8] J. Y. Wang, C. C. Yang, and Y. W. Kiang, "Numerical study on surface plasmon polariton behaviors in periodic metal-dielectric structures using a plane-wave-assisted boundary integral-equation method," Opt. Express, vol. 15, no. 14, pp. 9048-9062, Jul. 2007.

[9] F. Wooten, Optical Properties of Solids. New York: Academic, 1972.

[10] G. Sun, J. B. Khurgin, and R. A. Soref, "Practicable enhancement of spontaneous emission using surface plasmons," Appl. Phys. Lett., vol. 90, no. 11, p. 111107 , Mar. 2007.

[11] J. B. Khurgin, G. Sun, and R. A. Soref, "Enhancement of luminescence efficiency using surface plasmon polaritons: Figures of merit," J. Opt. Soc. Amer. B, Opt. Phys., vol. 24, no. 8, pp. 1968-1980, Aug. 2007.

[12] D. M. Yeh, C. Y. Chen, Y. C. Lu, C. F. Huang, and C. C. Yang, "Formation of various metal nanostructures with thermal annealing to control the effective coupling energy between a surface plasmon and an InGaN/GaN quantum well," J. Inst. Phys. Nanotechnol., vol. 18, no. 26, p. 265402, Jul. 2007. 